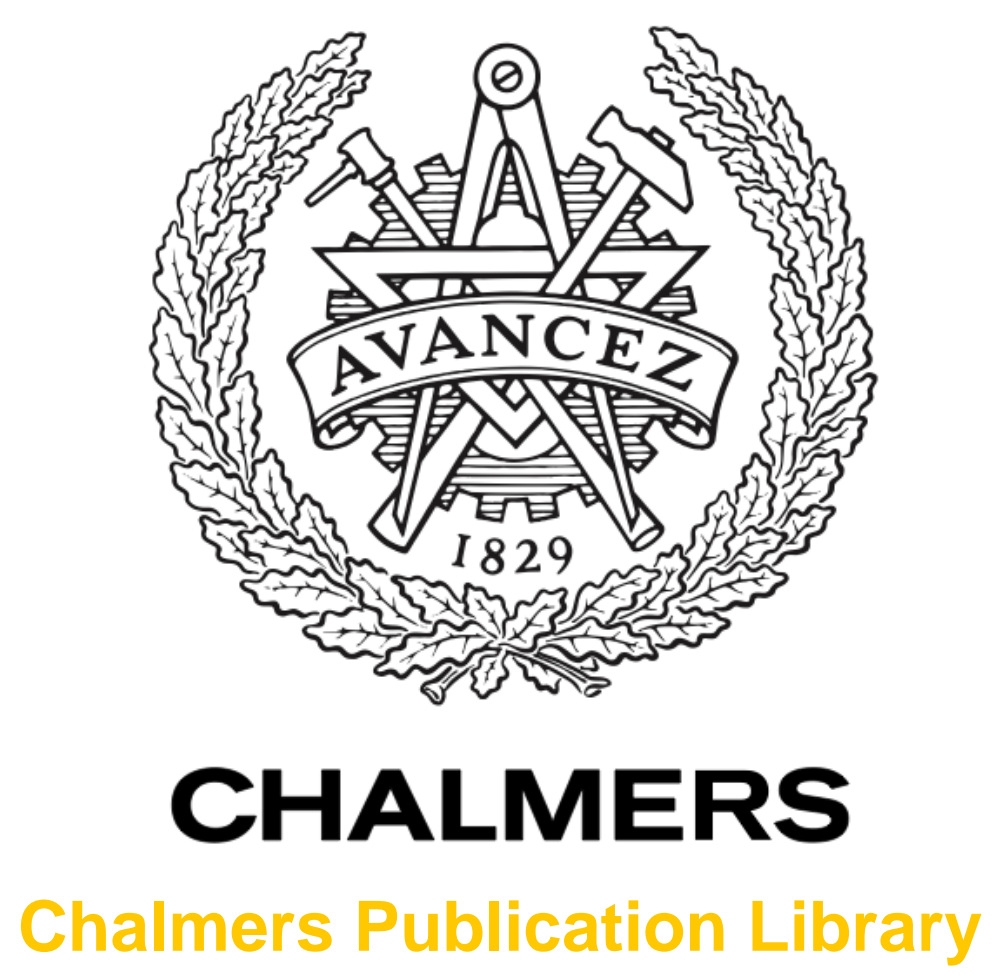

Microwave characterization of Ti/Au-graphene contacts

This document has been downloaded from Chalmers Publication Library (CPL). It is the author's version of a work that was accepted for publication in:

Applied Physics Letters (ISSN: 0003-6951)

Citation for the published paper:

Andersson, M. ; Vorobiev, A. ; Sun, J. (2013) "Microwave characterization of Ti/Augraphene contacts". Applied Physics Letters, vol. 103(17), pp. 173111.

http://dx.doi.org/10.1063/1.4826645

Downloaded from: http://publications.lib.chalmers.se/publication/185656

Notice: Changes introduced as a result of publishing processes such as copy-editing and formatting may not be reflected in this document. For a definitive version of this work, please refer to the published source. Please note that access to the published version might require a subscription.

Chalmers Publication Library (CPL) offers the possibility of retrieving research publications produced at Chalmers University of Technology. It covers all types of publications: articles, dissertations, licentiate theses, masters theses, conference papers, reports etc. Since 2006 it is the official tool for Chalmers official publication statistics. To ensure that Chalmers research results are disseminated as widely as possible, an Open Access Policy has been adopted.

The CPL service is administrated and maintained by Chalmers Library. 


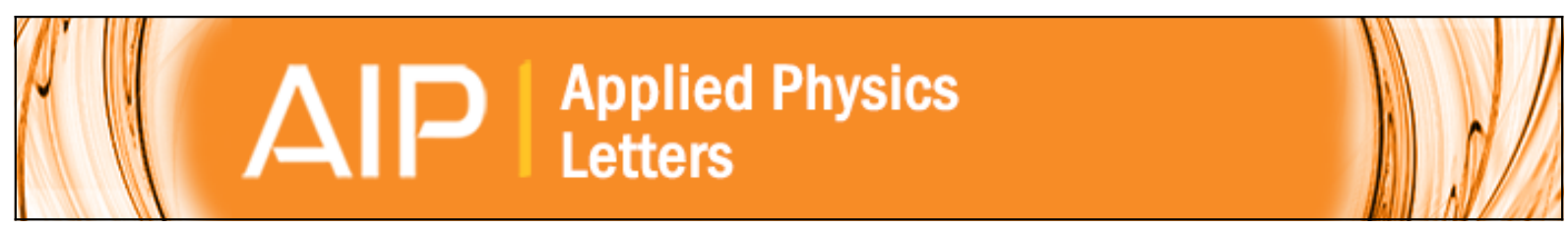

\section{Microwave characterization of Ti/Au-graphene contacts}

M. A. Andersson, A. Vorobiev, J. Sun, A. Yurgens, S. Gevorgian, and J. Stake

Citation: Applied Physics Letters 103, 173111 (2013); doi: 10.1063/1.4826645

View online: http://dx.doi.org/10.1063/1.4826645

View Table of Contents: http://scitation.aip.org/content/aip/journal/apl/103/17?ver=pdfcov

Published by the AIP Publishing

$\stackrel{A}{A} \mathbb{P} P$ Re-register for Table of Content Alerts

Create a profile.

Sign up today! 


\title{
Microwave characterization of Ti/Au-graphene contacts
}

\author{
M. A. Andersson, ${ }^{\text {a) }}$ A. Vorobiev, J. Sun, A. Yurgens, S. Gevorgian, and J. Stake \\ Department of Microtechnology and Nanoscience, Chalmers University of Technology, \\ SE-412 96 Gothenburg, Sweden
}

(Received 28 August 2013; accepted 10 October 2013; published online 24 October 2013)

\begin{abstract}
In this paper, we report on a microwave characterization of the interface between $\mathrm{Ti} / \mathrm{Au}$ contacts and chemical vapor deposition graphene using structures of Corbino geometry, with primary focus on extracting and modeling the capacitance associated with the contact region. It is found that with the current contact resistivity, $\rho_{c} \sim 10^{-6} \Omega \mathrm{cm}^{2}$, the contact capacitance, on the order $C_{c} \sim 1 \mu \mathrm{F} / \mathrm{cm}^{2}$, has a negligible effect on microwave transmission through the contact below $\sim 100 \mathrm{GHz}$. Finally, a parallel plate capacitance model for the contact is presented. (C) 2013 AIP Publishing LLC. [http://dx.doi.org/10.1063/1.4826645]
\end{abstract}

The extraordinary properties of graphene, a single layer of carbon atoms, ${ }^{1}$ are promising for a wide variety of applications, including microwave field effect transistors (FETs) ${ }^{2}$ and electrodes in optoelectronics. ${ }^{3}$ A key factor to achieve high performance devices is a complete understanding of the metalgraphene interface. This has been subject to both extensive theoretical work ${ }^{4,5}$ and experimental study for graphene prepared by exfoliation, ${ }^{4,6}$ chemical vapor deposition (CVD), ${ }^{7,8}$ and sublimation of $\mathrm{SiC}^{9}$ using the standard $\mathrm{DC}$ transfer length method (TLM) ${ }^{10}$ Generally, the sheet and contact resistances for relatively neutral exfoliated and CVD graphene are in the ranges of $2000-3000 \Omega /$ square and $750 \Omega \mu \mathrm{m}$, respectively, ${ }^{7}$ compared with values in the ranges of $200-300 \Omega$ /square and $<100 \Omega \mu \mathrm{m}$ for $\mathrm{SiC}$ graphene, ${ }^{9}$ with carrier concentration $n \sim 10^{13} \mathrm{~cm}^{-3}$ due to charge transfer from the substrate.

Efforts to characterize the microwave response are mainly limited to the graphene sheet resistance, which has been found to show no frequency dependence up to $110 \mathrm{GHz}$ so far. ${ }^{11,12}$ Conventional metal-semiconductor contacts have a capacitance associated with the barrier at the interface, which has been included in an extended TLM analysis. ${ }^{13}$ Although the metal-graphene contact is suggested to induce no barrier, ${ }^{6}$ still a contact capacitance, $C_{c}$, in parallel to the contact resistance, $R_{c}$, has been experimentally observed. ${ }^{14,15}$ The high capacitance is explained to stem from polymer residuals at the metal-graphene interface, which also results in a high contact resistance. ${ }^{14}$ On the other hand, using a complex equivalent circuit and an optimization procedure has led to unrealistic $C_{c}$ values with a frequency dependence ranging over several orders of magnitude. ${ }^{15}$

In this paper, we use the reflection coefficient of circular TLM (CTLM) structures, to avoid edge effects in the current flow, and study the RF response of a metal-graphene contact up to $20 \mathrm{GHz}$. A capacitive response interpreted to be that of a clean metal-graphene interface is deduced. Two methods are used in the analysis of the measurements. The value of $C_{c}$ is extracted both from a global frequency curve fitting procedure and utilizing extrapolating at each frequency point. The results from both methods agree reasonably well

\footnotetext{
${ }^{\text {a) }}$ Author to whom correspondence should be addressed. Electronic mail: andmic@chalmers.se.
}

and together with the contact resistance a cut-off frequency for the contact ${ }^{13}$ is estimated, $f_{c}>100 \mathrm{GHz}$, below which the contact capacitance may be ignored. This is in contrast to previous reports indicating this capacitance to be important for the performance of future graphene electronics. ${ }^{14,15}$

Graphene in this work was grown by CVD on $50 \mu \mathrm{m}$ thick copper foils (99.995\% purity, Advent) with methane as the carbon precursor. The growth time and temperature were $5 \mathrm{~min}$ and $1000^{\circ} \mathrm{C}$, respectively. ${ }^{16}$ The graphene was subsequently transferred to high resistivity $(>10 \mathrm{k} \Omega \mathrm{cm}) \mathrm{n}$-type $\mathrm{Si}$ substrates with $300 \mathrm{~nm}$ thermally grown $\mathrm{SiO}_{2}$ for $\mathrm{DC}$ and $\mathrm{RF}$ characterization. In addition, as reference, graphene on insulating fused silica glass substrates was used. The transfer is performed using a $300 \mathrm{~nm}$ thick PMMA layer spun onto the graphene, which is supported by a plastic frame to ease handling. Mechanical separation of the graphene from the copper using $\mathrm{H}_{2}$ bubbles is performed. ${ }^{17}$ Finally, the PMMA is dissolved in acetone heated to $50^{\circ} \mathrm{C}$ for $10 \mathrm{~min}$.

CTLM structures were patterned using electron beam lithography, which gives excellent dimensional control important for subsequent extrapolation methods. In addition, to our experience, it yields a cleaner graphene surface as compared with a photolithographic process, when no $\mathrm{O}_{2}$ plasma descumming can be used. This is crucial for low resistance ohmic contacts to graphene. ${ }^{8}$ Contacts consisting of $10 \mathrm{~nm}$ $\mathrm{Ti} / 100 \mathrm{~nm} \mathrm{Au}$ were e-beam evaporated and lifted-off. The inner pad radius was constant at $a=50 \mu \mathrm{m}$ with the graphene gap to the outer pad $s=5-25 \mu \mathrm{m}$, all fabricated on the same sample. A micrograph of a finished $s=25 \mu \mathrm{m}$ CTLM structure on $\mathrm{SiO}_{2} / \mathrm{Si}$ is shown in Fig. 1. The magnified image illustrates transferred graphene virtually free from holes.

S-parameter measurements were performed with an Agilent N5230A network analyzer and $125 \mu \mathrm{m}$ pitch groundsignal-ground (GSG) probes in the frequency range 2-20 GHz. A short-open-load (SOL) calibration was performed on a calibration substrate (CS-5).

The measured DC resistance versus gap spacing is shown in Fig. 2. The total resistance is obtained from the measured raw data by the de-embedding of a reproducible series resistance originating from cables and probe-to-gold contact resistance. The data are corrected for the circular layout of the structures to fit linearly to ${ }^{10}$ 


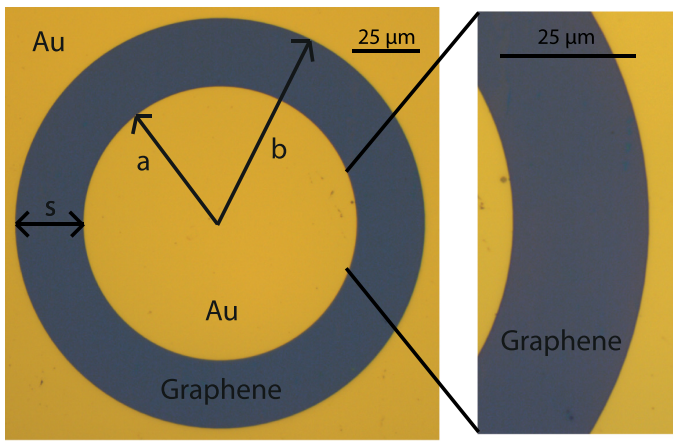

FIG. 1. Micrograph of a fabricated CTLM structure on $\mathrm{SiO}_{2} / \mathrm{Si}$ with $s=25 \mu \mathrm{m}$. Magnified area indicates the high quality of the transferred CVD grown graphene.

$$
R_{t o t}=\frac{R_{s h, \text { ring }}}{2 \pi a}\left[s+2 l_{x}\right] \times \underbrace{\frac{a}{s} \ln \left(\frac{b}{a}\right)}_{\mathrm{C}},
$$

with the dimensional quantities illustrated in Fig. 2. The contact resistance and graphene sheet resistance within the ring,

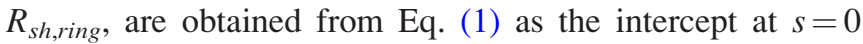
and from the slope, respectively, are presented in Table I. The sheet resistance of graphene under the contact, $R_{\text {sh,contact }}$, can be significantly altered due to the two-dimensional nature of graphene $e^{4,18}$ via charge transfer from the contact. ${ }^{5}$ The fact that $R_{\text {sh,ring }} \neq R_{\text {sh,contact }}$ yields $l_{x} \neq l_{t}$, where $l_{t}$ is the transfer length. Instead the transfer length and specific contact resistivity, $\rho_{c}$, will be estimated from the extracted capacitance of the contact. For comparison, a direct logarithmic fit to

$$
R_{\text {tot }}=2 R_{c}+\frac{R_{\text {sh, ring }}}{2 \pi} \ln \left(\frac{b}{a}\right)
$$

is performed and presented in Table I (data within brackets). The extracted contact resistance compares well to other results with $\mathrm{Ti} / \mathrm{Au}$ contacts on exfoliated or CVD grown graphene, originating from a clean graphene surface and a low evaporator pressure, $<5 \times 10^{-7}$ Torr. $^{19}$

Furthermore, the current-voltage (I-V) curve from back gating on the $\mathrm{SiO}_{2} / \mathrm{Si}$ sample reveals a p-type doping of the graphene. Fitting the measured transfer characteristics ${ }^{20}$ at the Dirac point, a residual carrier density $n_{0} \simeq 4 \times 10^{11} \mathrm{~cm}^{-2}$ is extracted, compared with the hole doping $p \sim 10^{12} \mathrm{~cm}^{-2}$

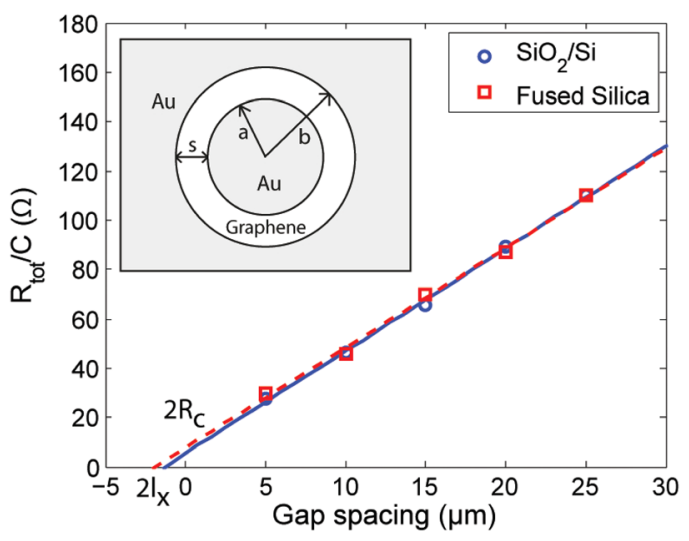

FIG. 2. DC CTLM extrapolation on $\mathrm{SiO}_{2} / \mathrm{Si}$ and silica glass. Inset: structure layout with relevant dimensions indicated.
TABLE I. Contact resistivity and ring sheet resistance from Eqs. (1) and (2) (data within brackets) based on two sets of CTLM structures each.

\begin{tabular}{lcl}
\hline \hline Substrate & $R_{c} \times W(\Omega \mu \mathrm{m})$ & \multicolumn{1}{c}{$R_{\text {sh }}(\Omega /$ square $)$} \\
\hline $\mathrm{SiO}_{2} / \mathrm{Si}$ & $860(890) \pm 150$ & $1300(1280) \pm 100$ \\
Silica glass & $1230(1250) \pm 40$ & $1270(1240) \pm 40$ \\
\hline \hline
\end{tabular}

$\left(\left|E_{F}\right| \sim 0.1 \mathrm{eV}\right)$ at zero gate bias. This explains the relatively low sheet resistances obtained and the low contact resistance as compared with previous $\mathrm{Ti} / \mathrm{Au}$ contacts reported on CVD graphene. $^{7}$

In the small-signal models of Corbino structures on both fused silica and $\mathrm{SiO}_{2} / \mathrm{Si}$ substrates, the complex graphene sheet impedance is generally written as $Z_{g r}=\left(\sigma_{g r}\right)^{-1}$ $=R_{g}+j \omega L_{k},{ }^{21}$ where $\sigma_{g r}$ is given by

$$
\sigma_{g r}=-j \frac{q^{2} k_{B} T}{\pi \hbar^{2}(\omega-j \tau)}\left[\frac{\mu_{c}}{k_{B} T}+2 \ln \left(1+e^{-\frac{\mu_{c}}{k_{B} T}}\right)\right] .
$$

In Eq. (3), $\omega=2 \pi f, q$ is the electron charge, $\hbar$ is the Planck constant, and $k_{B}$ Boltzmann constant. Furthermore, $\tau$, the carrier scattering time, is used as a fitting parameter to match the slightly differing sheet resistances of individual structures, likely originating from random defects induced during the transfer process. Once $\tau$ is known the kinetic inductance $L_{k}$ is calculated straightforwardly. The model is valid at room temperature, i.e., $T=300 \mathrm{~K}$. Finally, since no gate DC field is applied and as the graphene sheet has low carrier concentration, we assume for simplicity that the chemical potential $\mu_{c} \simeq 0$.

The gap dependences of the real and imaginary part of impedance for CTLM structures on insulating fused silica glass are shown in Fig. 3, where the symbols and solid lines correspond to measured and model fits, respectively. The real part is frequency independent, with a value in good agreement with the DC measurements. This consists of the graphene ring resistance, $R_{g}$, contact resistances for the inner patch, $R_{c}$, and the outer ground plane, $R_{c g p l}$ $\simeq R_{c} /\left(\frac{2 \pi b l_{t}}{2 \pi a l_{t}}\right)=R_{c} /\left(\frac{b}{a}\right)$, see Fig. 4. This is valid under the assumption $l_{t} \ll a, b$. The capacitive response of the imaginary part is partly explained by the parasitic pad-to-pad capacitance, $C_{\text {par }}$. It is extracted from method of moments

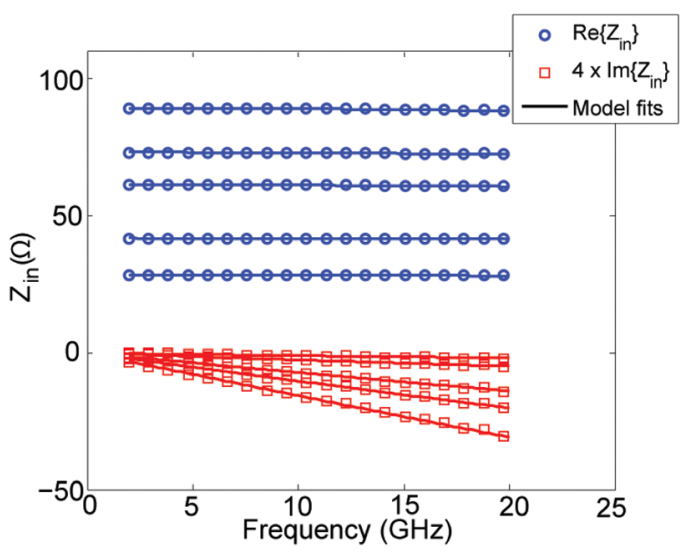

FIG. 3. Graphene on fused silica. Real part (circles), from bottom to top $s=5-25 \mu \mathrm{m}$, and imaginary part (squares), from top to bottom $s=5-25 \mu \mathrm{m}$. N.B. $\operatorname{Im}\left\{Z_{\text {in }}\right\}$ scaled $\times 4$. 


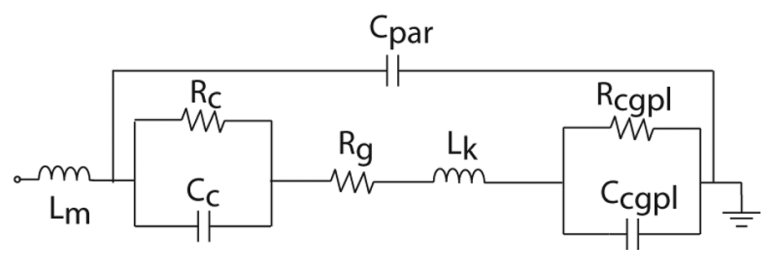

FIG. 4. Equivalent circuit of graphene Corbino structure on fused silica substrate, where $R_{c}\left(C_{c}\right)$ and $R_{c g p l}\left(C_{c g p l}\right)$ represent inner patch (outer ground plane), respectively.

simulations of identical structures excluding the graphene sheet. The remaining part originates from the capacitance associated with the metal-graphene inner and outer contacts, $C_{c}$ and $C_{c g p l}$, respectively. In a similar manner to the resistances, we assume $C_{c g p l} \simeq C_{c} \times\left(\frac{2 \pi b l_{t}}{2 \pi a l_{t}}\right)=C_{c} \times\left(\frac{b}{a}\right)$.

The final component in the circuit is the series inductance originating as a sum from inner pad and outer groundplane, $L_{m}=13 \mathrm{pH}$, chosen to be consistent with the fit of the extrapolated contact reactance $X_{c}$. The remaining curve fitting parameters are $\tau$ and the contact capacitance $C_{c}$, while the contact resistance was fixed at the DC value $R_{c}=3.9 \Omega$. The extracted values for the inner contact capacitance of one set of CTLM structures with varying gap are presented in Table II (a second set yielded values in the same range), together with the value extracted from extrapolated data, see below.

Alternatively, for confirmation of the results, real and imaginary parts of CTLM structure impedance can be extrapolated versus gap spacing at each frequency. Both the real and imaginary parts are corrected similarly to what is shown in Eq. (1) for the DC resistance. The reactance of the graphene structures becomes inductive at smaller gaps and the best fit at zero gap, after de-embedding the inductance, is to a parallel RC model given by the parameters $R_{c}=3.9 \Omega$ and $C_{c}=310 \mathrm{fF}$.

The measured impedance of graphene CTLM structures on conductive $\mathrm{SiO}_{2} / \mathrm{Si}$ substrates together with the model fits is shown in Fig. 5. The equivalent circuit now includes the oxide capacitance, $C_{o x}$, and resistance, $R_{o x}$, and also the conductive silicon surface via $R_{S i}$. The main signal path through the $\mathrm{Si}$ is in parallel to the graphene after traversing the contacts, as indicated in Fig. 6. Separate identical Corbino structures excluding the graphene sheet were measured and used in the curve fitting routine to model this behavior. The result is a signal leakage path which distorts the logarithmic extrapolation. Instead, an approximation using 2nd order polynomials was used. The resulting $C_{c}$ from curve fitting and extrapolation are summarized in Table II for one set of structures (another set yielded values in the same range), where $L_{m}=14 \mathrm{pH}$ is used. The value of $R_{c}$ from RF measurements for consistent values of $C_{c}$ for the different gaps, $R_{c}=6.2 \Omega$, is slightly higher compared with the DC value $\sim 3 \Omega$.

TABLE II. Capacitance from curve fitting and extrapolation on fused silica (first row) and $\mathrm{SiO}_{2} / \mathrm{Si}$ (second row).

\begin{tabular}{lcccccc}
\hline \hline Gap & $5 \mu \mathrm{m}$ & $10 \mu \mathrm{m}$ & $15 \mu \mathrm{m}$ & $20 \mu \mathrm{m}$ & $25 \mu \mathrm{m}$ & Extrapolation \\
\hline$C_{c, F S}(f F)$ & 380 & 370 & 400 & 390 & 310 & 310 \\
$C_{c, \mathrm{SiO}_{2}}(f F)$ & 450 & 490 & 430 & 430 & 440 & 390 \\
\hline \hline
\end{tabular}

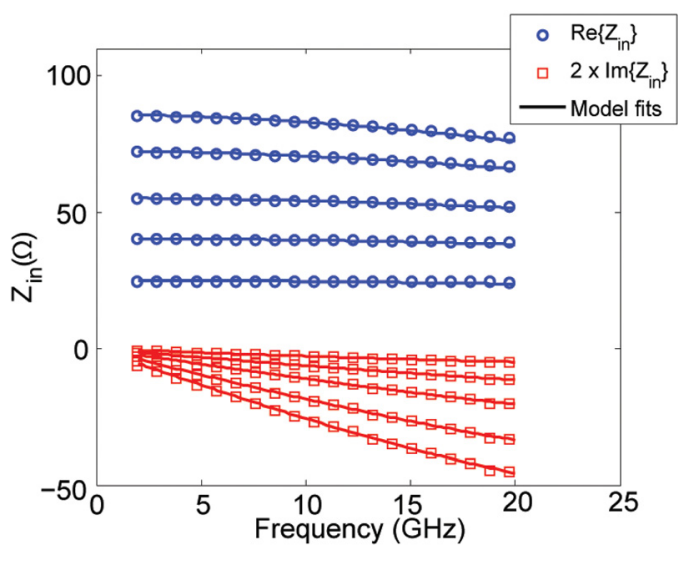

FIG. 5. Graphene on $\mathrm{SiO}_{2} / \mathrm{Si}$. Real part (circles), from bottom to top $s=5-25 \mu \mathrm{m}$, and imaginary part (squares), from top to bottom $s=5-25 \mu \mathrm{m}$. N.B. $\operatorname{Im}\left\{Z_{\text {in }}\right\}$ scaled $\times 2$.

We suggest a purely geometrical model for the contact capacitance, i.e., a parallel plate capacitor model, with a metal graphene separation distance, $d_{m-g}$, and a transfer length, $l_{t}$, according to Fig. 7 and given by

$$
C_{c}=C_{g e o}=\frac{\varepsilon_{0} \times A_{c}}{d_{m-g}} \simeq \frac{\varepsilon_{0} \times 2 \pi a l_{t}}{d_{m-g}},
$$

where $\varepsilon=\varepsilon_{0}$ is assumed. The graphene-metal distance, $d_{m-g}$, is to be considered an average value. At specific points, the separation is on the order of $\sim 2 \AA$, the theoretical binding distance between $\mathrm{Ti}$ and graphene. ${ }^{22}$ This is in line with the fact that even freestanding graphene exhibits ripples out of plane on the order of $1 \mathrm{~nm}$ (Ref. 23) and that graphene on substrates will have similar corrugations reflecting the underlying surface. ${ }^{24}$ This capacitance should be considered a property of a clean metal-graphene interface. Thus, e.g., $\mathrm{Pd} / \mathrm{Au}$ contacts are expected to show similar $C_{c}$ fixed by substrate roughness. On the contrary, the higher $C_{c}$ values previously reported are most likely results of other mechanisms, such as polymer residuals from photolithography. ${ }^{14}$ Since $C_{c}$ is an out-of plane capacitance it will be in series with the quantum capacitance of graphene ${ }^{25}$ associated with the transfer region area. However, $C_{q}$ is several times larger than $C_{g e o}$ and is thus neglected in the following analysis.

Using Eq. (4) together with the extracted contact capacitance estimations of $l_{t}$ and $d_{m-g}$ are possible. These will be given by intervals since the ratio $l_{t} / d_{m-g}$ appears in Eq. (4). Furthermore, the specific contact resistivity, given by $\rho_{c}=R_{c} \times A_{c}$, can be calculated. Previous reports of the transfer length of metal-graphene contacts were in the range

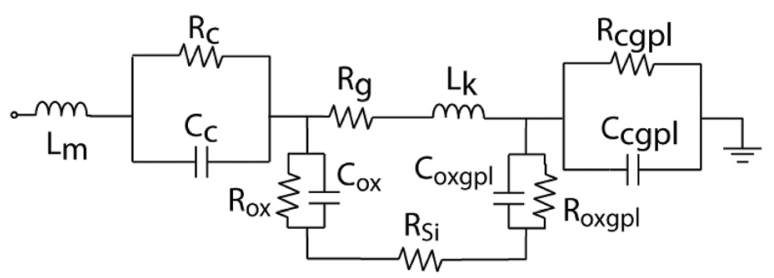

FIG. 6. Equivalent circuit of graphene Corbino structure on $\mathrm{SiO}_{2} / \mathrm{Si}$ substrate, where $R_{o x} / C_{o x}$ and $R_{S i}$ represent the lossy oxide and conductive Si surface, respectively. 


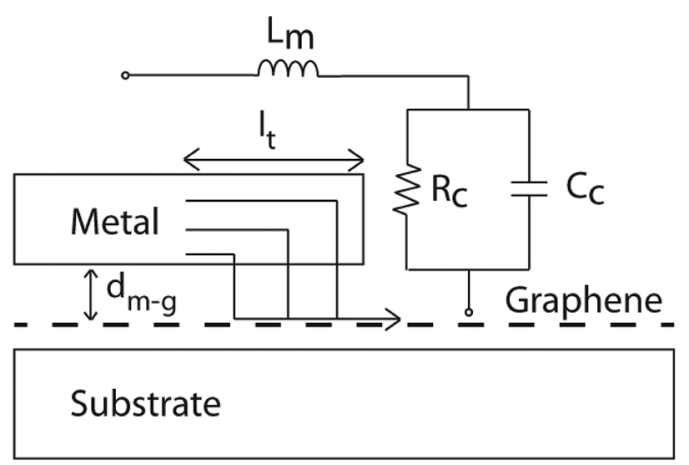

FIG. 7. Physical outline of the contact interface with the corresponding lumped circuit model. The mean separation $d_{m-g}$ results in a contact capacitance $C_{c}$.

$l_{t}=50-250 \mathrm{~nm}^{4}$ This corresponds to an interval of the separation $d_{m-g}=0.4-1.9 \mathrm{~nm}$ using an average value $C_{c}$ $=360 \mathrm{fF}\left(C_{c} \sim 1 \mu \mathrm{F} / \mathrm{cm}^{2}\right)$ for fused silica, in line with the previous discussion. Additionally, transfer length and separation can be used as direct optimization variables using exact expressions for the transfer region areas. Similarly, the range of values yielding the optimum fit is within $l_{t}=90-270 \mathrm{~nm}$ and $d_{m-g}=0.67-2.09 \mathrm{~nm}$. The fact $l_{t}<l_{x} \sim 1 \mu \mathrm{m}$ (cf. Fig. 2) suggests $R_{\text {sh,contact }}>R_{\text {sh,ring }}{ }^{10}$ possible by electron transfer from the Ti contact ${ }^{5}$ counteracting the p-type graphene under the contact making it more neutral. The estimated specific contact resistivity is $\rho_{c}=0.6-3 \times 10^{-6} \Omega \mathrm{cm}^{2}$. The crossover frequency above which the capacitance influences microwave transmission through the contact is given by a cut-off frequency similar to that for the TLM model, ${ }^{13}$ or the inverse of the RC time constant, $f_{c}=\left(2 \pi \rho_{c} \times C_{c, \mathrm{~F} / \mathrm{cm}^{2}}\right)^{-1}$ $=\left(2 \pi R_{c} \times C_{c}\right)^{-1} \sim 125 \mathrm{GHz}$. For the $\mathrm{SiO}_{2} / \mathrm{Si}$ sample, an average value of $C_{c}=440 \mathrm{fF}$ yields at least $f_{c} \sim 60 \mathrm{GHz}$ using $R_{c}=6.2 \Omega$. The ranges of $l_{t}$ and $d_{m-g}$ are similar to the fused silica case. The lower expected $\rho_{c}$ values ${ }^{4,7}$ with, e.g., Pd based contact would then make an equal contact capacitance even less influential on the microwave signal transmission properties compared with the Ti/Au case.

In conclusion, we reported on the high-frequency response of metal-graphene ohmic contacts. A geometrical model for the capacitance was suggested. The $C_{c}$ values are considerably lower compared with previous reports, ${ }^{14,15}$ implying the capacitance to have negligible influence on contact signal transmission at the current $\rho_{c}$ levels. The use of alternative contact metals is a subject planned for future study with the aim to further reduce the contact resistance and the microwave frequency dispersion.

This work was supported by the Swedish Research Council Project 2012-4978 and the Knut and Alice Wallenberg Foundation (KAW).

${ }^{1}$ K. S. Novoselov, A. K. Geim, S. V. Morozov, D. Jiang, V. Zhang, S. V. Dubonos, I. V. Grigorieva, and A. A. Frisov, Science 306, 666 (2004).

${ }^{2}$ F. Schwierz, Proc. IEEE 101, 1567 (2013)

${ }^{3}$ X. Kun, X. Chen, D. Jun, Z. Yanxu, G. Weiling, M. Mingming, Z. Lei, and J. Sun, Appl. Phys. Lett. 102, 162102 (2013).

${ }^{4}$ F. Xia, V. Perebeinos, Y.-M. Lin, Y. Wu, and P. Avouris, Nat. Nanotechnol. 6, 179 (2011).

${ }^{5}$ P. A. Khomyakov, G. Giovannetti, P. C. Rusu, G. Brocks, J. van den Brink, and P. J. Kelly, Phys. Rev. B 79, 195425 (2009).

${ }^{6}$ K. Nagashio and A. Toriumi, Jpn. J. Appl. Phys. Part 1 50, 070108 (2011). ${ }^{7}$ B.-C. Huang, M. Zhang, Y. Wang, and J. Woo, Appl. Phys. Lett. 99, 032107 (2011).

${ }^{8}$ A. Hsu, H. Wang, K. K. Kim, J. Kong, and T. Palacios, IEEE Electron Device Lett. 32, 1008 (2011).

${ }^{9}$ J. S. Moon, M. Antcliffe, H. C. Seo, D. Curtis, S. Lin, A. Schmitz, I. Milosavljevic, A. A. Kiselev, R. S. Ross, D. K. Gaskill, P. M. Campbell, R. C. Fitch, K.-M. Lee, and P. Asbeck, Appl. Phys. Lett. 100, 203512 (2012).

${ }^{10}$ D. K. Schröder, Semiconductor Material and Device Characterization (John Wiley, 2006).

${ }^{11}$ H. Skulason, H. Nguyen, A. Guermoune, V. Sridharan, M. Siaj, C. Caloz, and T. Szkopek, Appl. Phys. Lett. 99, 153504 (2011).

${ }^{12}$ W. Liu, V. Aguilar, Y. Hao, R. S. Ruoff, and N. P. Amitage, J. Appl. Phys. 110, 083510 (2011).

${ }^{13}$ H. H. Berger, Solid-State Electron. 15, 145 (1972).

${ }^{14}$ H. Skulason, H. Nguyen, A. Guermoune, M. Siaj, C. Caloz, and T. Szkopek, in Microwave Symposium Digest (MTT), IEEE MTT-S International, 2012.

${ }^{15}$ S. Moon, K. Jung, K. Park, H. J. Kim, C.-W. Lee, C.-W. Baik, and J. M. Kim, New J. Phys. 12, 113031 (2010).

${ }^{16}$ J. Sun, N. Lindvall, M. Cole, K. T. T. Angel, T. Wang, K. B. K. Teo, D. H. C. Chua, J. Liu, and A. Yurgens, IEEE Trans. Nanotechnol. 11, 255 (2012).

${ }^{17}$ C. J. Lockhart de la Rosa, J. Sun, N. Lindvall, M. T. Cole, Y. Nam, M. Löffler, E. Olsson, K. B. K. Teo, and A. Yurgens, Appl. Phys. Lett. 102, 022101 (2013).

${ }^{18}$ J. H. Klootwijk and C. E. Timmering, in Proceedings of the IEEE 2004 International Conference on Microelectronic Test Structures, 2004.

${ }^{19}$ S. Russo, M. F. Craciun, M. Yamamoto, A. F. Morpurgo, and S. Tarucha, Physica E 42, 677 (2010).

${ }^{20}$ S. Kim, J. Nah, I. Jo, D. Shahrjerdi, L. Colomobo, Z. Yao, E. Tutuc, and S. K. Banerjee, Appl. Phys. Lett. 94, 062107 (2009).

${ }^{21}$ G. Lovat, IEEE Trans. Electromagn. Compat. 54, 101 (2012).

${ }^{22}$ C. Gong, G. Lee, B. Shan, E.-M. Vogel, R. M. Wallace, and K. Cho, J. Appl. Phys. 108, 123711 (2010).

${ }^{23}$ J. C. Meyer, A. K. Geim, M. I. Katsnelson, K. S. Novoselov, T. J. Booth, and S. Roth, Nature 446, 60 (2007).

${ }^{24}$ C. H. Lui, L. Liu, K. F. Mak, G. W. Flynn, and T. F. Heinz, Nature 462, 339 (2009).

${ }^{25}$ T. Fang, A. Konar, H. Xing, and D. Jena, Appl. Phys. Lett. 91, 092109 (2007). 